
licence (http://creativecommons.org/licenses/by/4.o/), which permits unrestricted re-use, distribution, and reproduction in any medium, provided the original work is properly cited.

doi:10.1017/So144686X18000132

\title{
Typologies of loneliness, living alone and social isolation, and their associations with physical and mental health
}

\author{
KIMBERLEY J. SMITH* and CHRISTINA VICTOR $\dagger+$
}

\begin{abstract}
The relationship between living alone, loneliness and social isolation, and how they are associated with health remain contentious. We sought to explore typologies based on shared experiences of loneliness, social isolation and living alone using Latent Class Analysis and determine how these groups may differ in terms of their physical and mental health. We used Wave 7 of the English Longitudinal Study of Ageing $\left(\mathrm{N}=7, \mathrm{O}_{2}\right.$; mean age $\left.=67.3\right)$ and responses to the University of California, Los Angeles (UCLA) loneliness scale, household composition, participation in social/societal activities plus frequency of contact with friends, family and relatives for the Latent Class Analysis. The optimal number of groups was identified using model-fit criteria. The socio-demographic characteristics of groups and health outcomes were explored using descriptive statistics and logistic regression. We identified a six-cluster typology: Group 1, no loneliness or isolation; Group 2, moderate loneliness; Group 3, living alone; Group 4, moderate isolation; Group 5 , moderate loneliness, living alone; and Group 6, high loneliness, moderate isolation (with high likelihood of living alone). Groups experiencing loneliness and/or isolation were more likely to report poorer physical and mental health even after adjusting for socio-demographic confounders, this was particularly notable for Group 6. Our results indicate that different typologies of living alone, loneliness and isolation can be identified using data-driven techniques, and can be differentiated by the number and severity of issues they experience.
\end{abstract}

KEY WORDS - isolation, loneliness, living alone, typologies, health, older adults.

* Department of Psychological Sciences, School of Psychology, University of Surrey, Guildford, UK.

$\dagger$ Institute of Health, Environment and Societies, Brunel University London, Uxbridge, UK.

$\$$ Department of Clinical Sciences, Brunel University London, Uxbridge, UK. 


\section{Introduction}

Are loneliness, social isolation and living alone the same or different? Researchers and theorists have long hypothesised that loneliness, social isolation and living alone are separate constructs despite overlapping features (Victor et al. 2000). The identification of typologies of loneliness and social isolation has long been of interest to researchers (Townsend 1980; Tunstall 1966; Weiss 1973). Identifying typologies tells us how these concepts are related and can help us to identify better those people who may be at high risk of the adverse consequences of loneliness and social isolation such as poor health and wellbeing (Heinrich and Gullone 2006; Luanaigh and Lawlor 2008; Valtorta et al. 2016b). Andersson (1986) proposed a four-fold typology to summarise the relationship between loneliness and social isolation: neither lonely nor isolated, isolated but not lonely, lonely but not isolated, and both lonely and isolated. However, little research has explicitly examined whether these typologies may reflect the lived experience of older adults nor how different typologies might link with physical and mental health.

\section{The concepts of loneliness, social isolation and living alone}

Loneliness has been conceptualised in a number of different ways: as a discrepancy between a persons desired and perceived quality and quantity of social relationships (Walton et al. 1991), a perceived deprivation in social contact (Townsend 1980), perceived social isolation (Hawkley and Cacioppo 2010) or a lack of people with whom to share emotional and social experiences (Rook 1984). On the other hand, social isolation has been conceptualised as the measure of a person's integration and meaningful communication with their community, family and friends (Victor et al. 200o), the objective experience of being alone (Hawkley and Cacioppo 2010), a lack of meaningful social ties (Lubben and Gironda 2003) or a lack of integration with social networks (Rook 1984). Whilst these definitions suggest conceptual overlap, research shows there is only a moderate association between loneliness and social isolation (Golden et al. 2009).

Social isolation and loneliness can also be theoretically differentiated from living alone, a simple enumeration of household size (Victor et al. 200o). The evidence for the relationship of loneliness and social isolation with living alone is mixed, with studies variously reporting no association between loneliness (Zebhauser et al. 2014) or social isolation (Larson, Zuzanek and Mannell 1985) and living alone, high levels of loneliness/isolation amongst those living alone (Tunstall 1966) or that living alone is a strong risk factor for loneliness (Sundström et al. 2009). Despite possible 
theoretical differences, living alone has been included in measures of social isolation (Shankar et al. 2011 , 2013 ; Steptoe et al. 2013 b; Victor et al. 2000) rather than being evaluated as a potentially separate category.

\section{Loneliness, social isolation, living alone and health}

Existing theory and evidence suggest that a more nuanced examination of the relationships between these three concepts could be useful, especially in developing our understanding of the association of these issues with health and wellbeing outcomes. Previous qualitative research provides us with rich and meaningful data on the lived experiences of loneliness, social isolation and living alone. They indicate that loneliness and social isolation are complex individual experiences which have different perceived causes and consequences (Cloutier-Fisher, Kobayashi and Smith 2011; Dahlberg 2007). However, quantitative work has typically defined loneliness and social isolation in predetermined, binary and stringent ways (Shankar et al. 201 1, 2013; Steptoe et al. 2013b) that may not accurately encapsulate the complexity of these experiences.

Previous work argues that researchers, policy makers and practitioners need to appreciate how we define and measure loneliness, isolation and living alone so we can better identify how these experiences might be linked with important health and wellbeing outcomes (Perissinotto and Covinsky 2014; Valtorta and Hanratty 2012; Valtorta et al. 2016a).

Loneliness, living alone and social isolation are all independently associated with similar health outcomes such as depression (Alpass and Neville 2003; Dean et al. 1992; Hawthorne 2008), poorer cognitive functioning (Bassuk, Glass and Berkman 1999; Cacioppo and Hawkley 20o9; Zunzunegui et al. 2003), cardiovascular disease (Orth-Gomér, Rosengren and Wilhelmsen 1993; Valtorta et al. 2016b), poorer self-rated health (Kharicha et al. 2007; Lee et al. 2008; Nummela, Seppänen and Uutela 2011 ), worsened physical functioning (Iliffe et al. 2007; Kharicha et al. 2007; Perissinotto, Cenzer and Covinsky 2012) and lifestyle (Alpass and Neville 2003; Davis et al. 1990).

When researchers examine loneliness and social isolation with health simultaneously results are often mixed, with studies reporting loneliness and social isolation being associated with different health outcomes (Cornwell and Waite 2009; Elovainio et al. 2017; Holwerda et al. 2013; McHugh et al. 2017; Shankar et al. 2011; Steptoe et al. 2013b). However, there is a lack of work that examines how the experiences of living alone, loneliness and social isolation combine, and how these groups might be linked with health.

One way to determine whether meaningful typologies based on shared characteristics of social isolation, loneliness and living alone can be 
uncovered quantitatively is to use data-driven methods such as Latent Class Analysis (LCA) as a method to identify distinct sub-groups based on social relationships in older populations (Burholt, Dobbs and Victor 2017; Ellwardt, Aartsen and van Tilburg 2016). The first aim of our study was to identify groups of older people based on shared characteristics of loneliness, social isolation and living alone using LCA. The second aim of this study was to determine how each of these groups differed in terms of health and socio-demographic characteristics.

\section{Methods}

\section{Data-set and participants}

This study utilised data from Wave 7 of the English Longitudinal Study of Ageing (ELSA). ELSA is a longitudinal prospective cohort study of adults

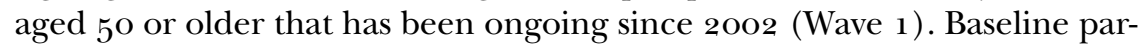
ticipants were sampled from eligible participants who had taken part in the Health Survey for England (HSE) in either 1998, 1999 or 2001 (Steptoe et al. $2013 a$ ). Core data collection for the ELSA study takes place every two years. As well as following-up baseline participants, refreshment sampling (i.e. sampling new participants from HSE) for ELSA has taken place at Waves 3, 4, 6 and 7. Our analysis utilises Wave 7, the most recently published wave of ELSA (data collection 2014-2015). A total of 8,249 people took part in this wave. We excluded people aged less than $5^{\circ}(\mathrm{N}=76)$, those who had a proxy answer on their behalf $(\mathrm{N}=597)$ or had incomplete data for indicators of loneliness, social isolation and living alone $(\mathrm{N}=544)$, giving an analytic sample of 7,032 (mean age $=67.3 ; 55 \%$ female) .

\section{Indicators of loneliness and social isolation}

The indicators of loneliness and social isolation used for our analysis were based upon previous work from ELSA exploring these topics. Our indicators of loneliness were the three questions from the three-item University of California, Los Angeles (UCLA) Loneliness Scale (Hughes et al. 2004): How often do you feel you lack companionship? How often do you feel left out? How often do you feel isolated? Responses were: hardly ever/ never, sometimes or always.

We used the social isolation index developed for use with ELSA (Shankar et al. 2011 ) based on the five items of:

1. Household composition (living alone, living in multi-person household). 
2. Participation in societal/social activities (not involved/involved). This included participation in any social/recreational activities (e.g. evening classes, social clubs and sports clubs), societal (e.g. political parties or tenants groups) or voluntary activities.

3. Communication with family (more than once a month/less than once a month/no family).

4. Communication with relatives (more than once a month/less than once a month/no family).

5. Communication with friends (more than once a month/less than once a month/no family).

\section{Socio-demographic characteristics}

We examined the following socio-demographic characteristics: age, sex (male/female), ethnicity (white/non-white), marital status (married/not married/divorced or separated/widowed), employment status (employed/ unemployed/retired), education (no qualifications/high school or collegelevel qualifications/higher education qualifications), net wealth minus pension (quintiles 1 and $2 /$ quintiles $3^{-5}$ ) - an indicator of wealth used in similar studies (Shankar et al. 2011 ).

\section{Health-related outcomes (physical and mental health)}

Functioning was assessed by calculating the number of issues with the following activities of daily living (ADLs): getting out of bed, dressing, walking, bathing, eating and/or using the toilet. Groups were categorised according to whether they experienced no issues or one or more issues with ADLs in line with previous work (Gale, Cooper and Aihie Sayer 2015).

Number of chronic conditions involved summing the number of chronic conditions people reported having from a list of 19 included in ELSA (for a list of conditions, see Dhalwani et al. 2016). Conditions were categorised as none, one, or two or more (multi-morbidity) (Dhalwani et al. 2017).

Self-rated health was assessed using a single item where people rated their own health on a five-point scale that ran from excellent to poor. Responses were dichotomised as excellent/very good/good or fair/poor in line with previous work (Badawi et al. 2013).

Depressive symptoms were measured using the eight-item Center for Epidemiological Studies Depression Scale (CES-D 8). This item measures the past week experience of eight indicators of depressive symptomatology (responses yes/no). For the sake of this analysis, the item on loneliness ('I have felt lonely') was removed in line with previous work that has examined loneliness and depressive symptoms using ELSA (Shankar et al. 2011 ). 


\section{Kimberley J. Smith and Christina Victor}

Responses were then categorised as high $\left(\geqslant_{4}\right)$ versus low $(<4)$ depressive symptoms in line with previous work ( $\mathrm{Au}$ et al. 2015; Hamer et al. 2009). However, as this cut-point was validated for the full eight-item scale we also ran sensitivity analyses with a cut-point of 3 (cut-point identified for the $10 \%$ with the highest depressive symptom score) to check our results.

\section{Analysis}

The indicators of loneliness, social isolation and living alone were grouped into meaningful groups using LCA (Latent Gold 5.0). LCA uses mathematical modelling to group categorical and/or continuous variables into classes (in this paper referred to as groups) based on likelihood estimates (Hagenaars and McCutcheon 2002; McCutcheon 1987; Vermunt and Magidson 2002). These classes represent groupings of data where people responded similarly to our indicators of loneliness, social isolation and living alone. To determine the optimal number of groups to derive we compared model fit from one to ten groups (run with 10,000 iterations). We determined the best model fit by examining the Bayesian Information Criteria (BIC), log-likelihood ratio (LLR), $p$-value and number of parameters. The model that demonstrated parsimony between the lowest number of parameters, highest $p$-value, and lowest BIC and LLR was chosen.

Once the optimal number of groups was identified, we examined the latent (previously unobserved) shared loneliness, social isolation and living alone characteristics that defined each group. Groups were then compared on socio-demographic characteristics and health-related outcomes using chisquared analysis for categorical variables and one-way analysis of variance for continuous variables. We then ran logistic regression analyses to examine the relationship between group membership and each health and wellbeing outcome (issues with ADLs, self-rated health, multi-morbidity and depressive symptoms) by comparing all groups who reported loneliness, isolation and/ or living alone to a reference group that was neither lonely nor isolated. Logistic regression analyses were first run unadjusted and then adjusted for socio-demographic characteristics (age, sex, marital status, wealth, employment and education). All analyses were conducted with SPSS 23.o.

\section{Results}

LCA: identifying groups based on shared loneliness and isolation characteristics

The LCA showed that the lowest BIC value was demonstrated by the sevengroup model (see Table 1), although the value for the six-group model was 
T A B LE 1. Model fit based on the number of groups to which the data are fitted

\begin{tabular}{lcccc}
\hline Number of groups & BIC & $p$ & Number of parameters & LLR \\
\hline 1 & $70,900.2900$ & $<0.001$ & 14 & -35388.1374 \\
2 & $65,181.5489$ & $<0.001$ & 26 & -32475.6175 \\
3 & $64,367.2683$ & 0.96 & 38 & -32015.3278 \\
4 & $64,070.8210$ & 1.00 & 50 & -31813.9548 \\
5 & $63,900.8480$ & 1.00 & 62 & -31675.8190 \\
6 & $63,789.9953$ & 1.00 & 74 & -31567.2433 \\
7 & $63,783.0452$ & 1.00 & 86 & -31510.6189 \\
8 & $63,829.5216$ & 1.00 & 98 & -3148.7077 \\
9 & $63,862.0824$ & 1.00 & 110 & -31443.8387 \\
10 & $63,899.1949$ & 1.00 & 122 & $-31409.245^{6}$ \\
\hline
\end{tabular}

Notes: BIC: Bayesian Information Criteria. LLR: log-likelihood ratio. Lower numbers for indicators of model fit (BIC and LLR) indicate better model fit. Higher $p$-values also indicate better model fit. The best match between model fit and $p$-values that results in the lowest number of parameters represents the optimum number of groups.

comparable. As the number of groups increased, the LLR indicated that model fit improved ( see Table 1), however, the number of parameters indicated that fewer groups was preferable whilst the $p$-value indicated any model fit from three groups onwards had a good fit. We used the sixgroup model as it demonstrated the greatest parsimony between the four model-fit indicators (for model-fit statistics, see Table 1 ).

The six groups identified differed in terms of their likelihood to exhibit different loneliness and living alone characteristics (see Table 2) which we characterised as:

- Group 1: no loneliness or isolation ( $46.97 \%$ of sample).

- Group 2: moderate loneliness (17.19\% of sample).

- Group 3: living alone ( $11.79 \%$ of sample).

- Group 4: moderate isolation ( $11.17 \%$ of sample).

- Group 5: moderate loneliness, living alone (9.03\% of sample).

- Group 6: high loneliness, moderate isolation (with high likelihood of living alone) $(3.85 \%$ of sample).

When differentiating groups based on loneliness, those groups with low loneliness were identified by a high likelihood of responding 'hardly ever or never' to loneliness questions. Those groups with moderate loneliness were identified by a high likelihood of responding 'sometimes' to loneliness questions. Finally, those groups with high loneliness were identified by a high likelihood of responding 'always' to loneliness questions. When differentiating groups based on isolation, low isolation was indicated by a high 
T A B LE 2. Characteristics of the six groups (classes) uncovered with Latent Class Analysis

\begin{tabular}{|c|c|c|c|c|c|c|}
\hline & Group 1 & Group 2 & Group 3 & Group 4 & Group 5 & Group 6 \\
\hline$\%$ & $4^{6.97}$ & 17.19 & 11.79 & 11.17 & 9.03 & 3.85 \\
\hline \multicolumn{7}{|l|}{ Lacks companionship: } \\
\hline Hardly ever or never & 0.936 & 0.333 & 0.706 & 0.907 & 0.072 & 0.003 \\
\hline Sometimes & 0.064 & 0.635 & 0.291 & 0.093 & $0.73^{1}$ & 0.298 \\
\hline Always & $<0.001$ & 0.032 & 0.003 & $<0.001$ & 0.198 & 0.699 \\
\hline \multicolumn{7}{|l|}{ Feels left out: } \\
\hline Hardly ever or never & 0.923 & 0.145 & 0.947 & 0.932 & 0.204 & $<0.001$ \\
\hline Sometimes & 0.077 & 0.827 & 0.053 & o.o68 & 0.778 & 0.237 \\
\hline Always & $<0.001$ & 0.029 & $<0.001$ & $<0.001$ & 0.018 & 0.762 \\
\hline \multicolumn{7}{|l|}{ Feels isolated: } \\
\hline Hardly ever or never & 0.963 & 0.246 & 0.964 & 0.894 & 0.260 & 0.002 \\
\hline Sometimes & 0.037 & 0.709 & 0.036 & 0.106 & o.698 & 0.225 \\
\hline Always & $<0.001$ & 0.046 & $<0.001$ & $<0.001$ & 0.042 & 0.774 \\
\hline \multicolumn{7}{|l|}{ Lives alone: } \\
\hline No & 0.996 & 0.918 & 0.098 & 0.918 & 0.142 & 0.492 \\
\hline Yes & 0.004 & 0.082 & 0.902 & 0.082 & $0.85^{8}$ & $0.5^{08}$ \\
\hline \multicolumn{7}{|l|}{ Contact with child: } \\
\hline Frequent contact & 0.885 & 0.853 & 0.735 & $0.75^{6}$ & 0.683 & $0.75^{1}$ \\
\hline Infrequent contact & 0.002 & 0.041 & 0.008 & $0.14^{1}$ & 0.023 & 0.061 \\
\hline No children & 0.113 & 0.106 & $0.25^{6}$ & 0.103 & 0.294 & 0.189 \\
\hline \multicolumn{7}{|l|}{ Contact with relatives: } \\
\hline Frequent contact & 0.829 & 0.724 & 0.770 & 0.482 & 0.724 & 0.673 \\
\hline Infrequent contact & 0.122 & 0.211 & 0.132 & 0.428 & $0.15^{0}$ & 0.246 \\
\hline No relatives & 0.049 & 0.064 & 0.099 & o.og1 & 0.126 & 0.081 \\
\hline \multicolumn{7}{|l|}{ Contact with friends: } \\
\hline Frequent contact & 0.957 & 0.760 & 0.963 & 0.545 & 0.925 & 0.682 \\
\hline Infrequent contact & 0.034 & $0.14^{1}$ & 0.019 & 0.230 & 0.029 & 0.069 \\
\hline No friends & 0.009 & o.o99 & 0.018 & 0.226 & 0.046 & 0.249 \\
\hline \multicolumn{7}{|c|}{ Social/societal involvement: } \\
\hline Not involved & 0.171 & 0.318 & 0.226 & $0.45^{8}$ & 0.240 & 0.459 \\
\hline Involved & 0.829 & 0.682 & 0.775 & 0.542 & 0.760 & $0.54^{1}$ \\
\hline
\end{tabular}

Notes: Group 1: no/low loneliness and isolation. Group 2: moderate loneliness. Group 3: lives alone. Group 4: moderate isolation. Group 5: moderate loneliness, lives alone. Group 6: high loneliness and moderate isolation. Results shown are likelihood estimates of members within each group responding in a particular way to each question (e.g. 0.171 indicates a $17.1 \%$ likelihood of giving that response/meeting that criteria). Results are rounded up to three decimal places and so not all results may add up to 100 .

likelihood of frequent contact with friends, children and family plus having social/societal involvement. Groups with moderate isolation were identified by having a higher likelihood of reporting positively to one or two of the social isolation indicators such as indicating less-frequent contact with 
friends, relatives or children plus reporting no social/societal involvement. High social isolation would be indicated by a higher likelihood of responding positively to all indicators of social isolation.

The first group, almost half of the sample, had a high likelihood of not being lonely, socially isolated or living alone. Groups 2-4 demonstrated a high probability of experiencing one of our three factors. Group 2 had the highest likelihood of reporting moderate feelings of loneliness (responding sometimes to feelings of loneliness). Group 4 had the highest likelihood of being isolated as measured by contact with family, friends and children when compared with other groups. Group 3 had a high likelihood $(90 \%)$ of living alone but a low likelihood of exhibiting loneliness or other isolation characteristics. Groups 5 and 6 demonstrated combinations of our three indicators. The fifth group showed a high likelihood of reporting moderate loneliness and living alone but a high likelihood of frequent contact with people and social/societal involvement and the highest likelihood of having no children $(29 \%)$ or relatives $(13 \%)$. The sixth group was most likely to report always being lonely, and also had a relatively high likelihood $(51 \%)$ of living alone and not being involved in social/societal activities $(46 \%)$. While not as high as Group 4, this group also demonstrated a greater likelihood of having less frequent contact with relatives $(25 \%)$ and a relatively high likelihood of having no children $(19 \%)$ and the highest likelihood of having no friends $(25 \%)$.

\section{Comparing groups on socio-demographic and health-related outcomes}

We firstly compared groups on socio-demographic characteristics (see Table 3), and there were significant differences for age, gender, marital status, net wealth, employment and education between the six groups. In terms of age, Groups 3 and 5 , both characterised by a high probability of living alone, were the oldest, the most likely to be retired and had the highest proportions of widows. Two of the three groups that had the highest proportion of married and employed participants had been characterised by a low likelihood of being lonely (Groups 1 and 4 ). However, the group that was characterised by moderate loneliness also had a high proportion of married and employed respondents (Group 2). Furthermore, the group characterised by experiencing no issues with loneliness or social isolation (Group 1) were the best educated. Those groups with the highest proportions of females were the groups most likely to live alone (Groups 3, 5 and 6) whilst the group with the highest proportion of males was the group that reported moderate isolation (Group 4). The group that had the highest proportion of respondents in the two lowest wealth quintiles 
TA B L E 3. Associations of group membership with socio-demographics, physical health and mental health

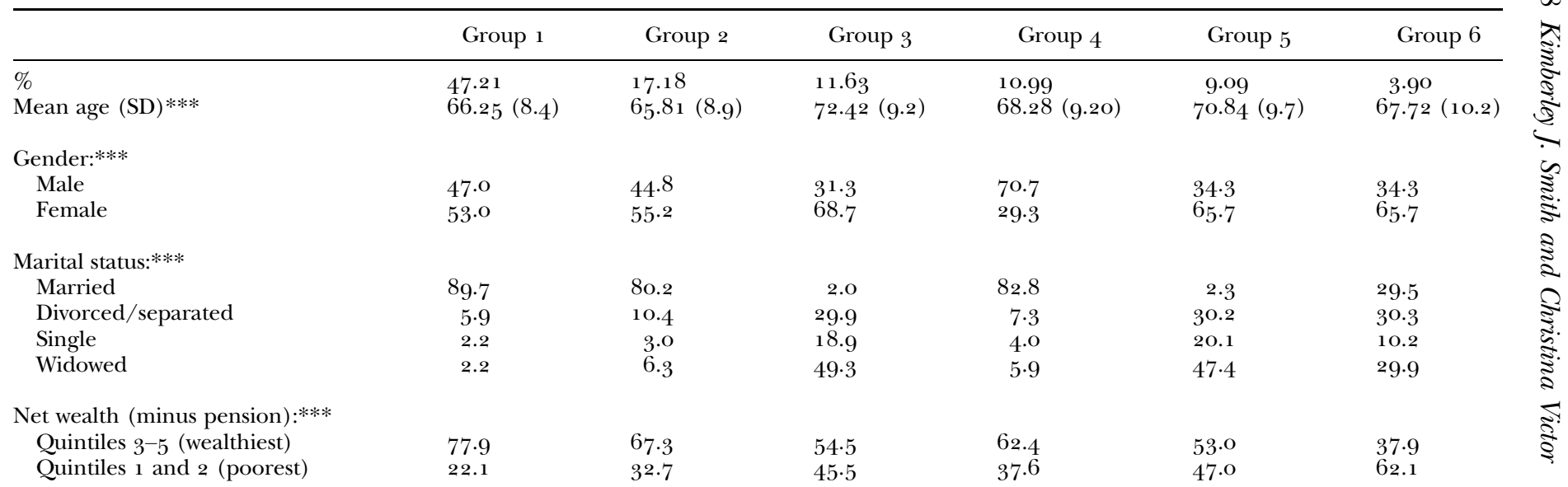

Educational qualifications:***

Degree or higher education

High school/college qualifications

$37.8 \quad 29.8$

46.9

48.9

$29 \cdot 7$

No qualifications

15.9

21.3

43.2

32.1

44.9

23.0

26.0

20.2

27.1

$44 \cdot 9$

$49 \cdot 0$
30.8

Employment:***

Employed (full/part/self)

Unemployed

37.8

37.0

$5 \cdot 9$

18.1

$5 \cdot 5$
76.4

$33 \cdot 7$

8.9

$57 \cdot 4$

20.4

8.8
70.8

20.1

$5^{1 \cdot 4}$

70.8

5.8

Functioning:***

No issues with ADLs

One or more issues with ADLs

$\begin{array}{rr}90.2 & 79.4 \\ 9.8 & 20.6\end{array}$

85.6

8 o. 6

$79 \cdot 4$

20.6 
Self-rated health:***

Excellent/very good/good

Fair/poor

$84.5 \quad 68.0$

32.0

75.6

$24 \cdot 4$

72.9

$65 \cdot 3$

$4^{6.1}$

Number of chronic conditions:***

None

$33 \cdot 9$

One

40.8 $\quad 33.9$

31.9

$27 \cdot 4$

28.0

28.3

$43 \cdot 7$

$35 \cdot 2$

31.9

32.9

$34 \cdot 7$

53.9

Depressive symptoms:***

Low

High

$96.8 \quad 81.6$

81.6
18.4

94.0

92.9

7.1

24.0

27.9

20.9

25.6

$53 \cdot 5$

Notes: SD: standard deviation. ADL: activities of daily living. Group 1: no/low loneliness and isolation. Group 2: moderate loneliness. Group 3: lives alone.

Group 4: moderate isolation. Group 5: moderate loneliness, lives alone. Group 6: high loneliness and moderate isolation.

Significance levels: $* p<0.05, * * p<0.01, * * * p<0.001$. 
and the highest proportion of unemployment was Group 6 (high loneliness, moderate isolation, high likelihood of living alone).

There were also significant differences between the six groups for all four health and wellbeing outcomes: functioning (issues with ADLs), self-rated health, number of chronic conditions and depressive symptoms (see Table 3). The groups who reported isolation and/or loneliness (Groups 2, 4, 5 and 6) had the highest proportion of two or more issues with ADLs). This association was strongest in the group with both high loneliness and isolation (Group 5). All of the groups who experienced loneliness (Groups 2, 5 and 6) had a high likelihood of reporting fair/ poor self-rated health ( see Table 3 ) and this was most pronounced in the group characterised by high loneliness and social isolation. The three groups who had the highest proportion of multi-morbidity (two or more chronic conditions) were the group characterised by living alone (Group 3) and the two characterised by shared loneliness and social isolation (Groups 5 and 6). All groups experiencing isolation and/or loneliness were more likely to exhibit high depressive symptoms (Groups 2, 4, 5 and 6 ). This association was strongest in the group with high loneliness and isolation (Group 6).

We then ran a series of logistic regression analyses to determine the association between group membership and health and wellbeing, adjusting for socio-demographic characteristics. Crude and adjusted estimates are presented in Table 4. Group 1, no loneliness or isolation, was our reference category for both our crude and adjusted analyses. For all crude analyses, Groups 2-6 inclusive reported poorer outcomes on all four physical and mental health outcomes. All associations were attenuated after adjustment for Group 3 (lives alone). Furthermore, the associations between Group 4 with multi-morbidity was also attenuated after adjustment. The most pronounced associations between group and health was observed for high depressive symptoms, where all groups (except Group 3) were more likely than Group 1 to report high depressive symptoms, and this was particularly pronounced in the group with high loneliness and isolation (Group 6).

\section{Sensitivity analysis}

As the cut-point we used for our depressive symptom analysis $(\geqslant 4)$ was based on a cut-off validated for the eight-item scale, we re-ran our analysis with a cut-point of $\geqslant 3$ to see what effect this had on our results. For our cross-tabulation analysis, the association between group membership and depressive symptoms remained highly significant $\left(\chi^{2}(5, \mathrm{~N}=6,970)=691.9, \quad p<\right.$ o.001). For the proportions of high depressive symptoms, see Table A in the online supplementary material. 
T A B L E 4. Logistic regression analyses of association between group membership and physical and mental health

\begin{tabular}{|c|c|c|c|c|c|c|c|c|}
\hline \multirow[b]{2}{*}{ Group } & \multicolumn{2}{|c|}{ One or more issues with ADLs ${ }^{1}$} & \multicolumn{2}{|c|}{ Fair/poor self-rated health ${ }^{2}$} & \multicolumn{2}{|c|}{ Two or more chronic conditions ${ }^{3}$} & \multicolumn{2}{|c|}{ High depressive symptoms ${ }^{4}$} \\
\hline & $\begin{array}{l}\text { Unadjusted } \\
\text { OR (95\% CI) }\end{array}$ & $\begin{array}{c}\text { Adjusted } \\
\text { OR (95\% CI) }\end{array}$ & $\begin{array}{c}\text { Unadjusted } \\
\text { OR (95\% CI) }\end{array}$ & $\begin{array}{c}\text { Adjusted } \\
\text { OR (95\% CI) }\end{array}$ & $\begin{array}{c}\text { Unadjusted } \\
\text { OR (95\% CI) }\end{array}$ & $\begin{array}{c}\text { Adjusted } \\
\text { OR }(95 \% \text { CI })\end{array}$ & $\begin{array}{c}\text { Unadjusted } \\
\text { OR (95\% CI) }\end{array}$ & $\begin{array}{c}\text { Adjusted } \\
\text { OR }(95 \% \text { CI })\end{array}$ \\
\hline 1 & 1 & 1 & 1 & 1 & 1 & 1 & 1 & 1 \\
\hline 2 & $\begin{array}{l}2.4^{1} \\
\quad\left(1.99^{-2.93}\right)^{* * * *}\end{array}$ & $\stackrel{2.13}{\left(1.73^{-2.62}\right)^{* * *}}$ & $\begin{array}{l}2.67 \\
(2.26-3.15)^{* * *}\end{array}$ & $\begin{array}{l}2.3^{6} \\
\quad\left(1.9^{8}-2.82\right)^{* * * *}\end{array}$ & $\begin{array}{l}1.72 \\
\quad\left(1.47^{-2.01}\right) * * *\end{array}$ & $\begin{array}{l}1.89 \\
\quad\left(1.5^{6-2.28}\right) * * *\end{array}$ & $\frac{7 \cdot 45}{(5 \cdot 74-9 \cdot 67)^{* * *}}$ & $\begin{array}{l}6.60 \\
\quad(5.05-8.63) * * *\end{array}$ \\
\hline 3 & $\begin{array}{l}1.5^{\circ} \\
\quad\left(1.19^{-1.89}\right)^{* *}\end{array}$ & $\begin{array}{l}0.86 \\
\quad(0.62-1.19)\end{array}$ & $\begin{array}{l}1.68 \\
\left(1.39^{-2.03}\right) * * *\end{array}$ & $\begin{array}{l}1.03 \\
\quad\left(0.7^{8}-1.36\right)\end{array}$ & $\begin{array}{l}2.32 \\
\quad\left(1.9^{2}-2.79\right)^{* * *}\end{array}$ & $\begin{array}{l}0.94 \\
\quad(0.71-1.23)\end{array}$ & $\begin{array}{l}1.93 \\
\quad\left(1.35^{-2.77}\right) * * *\end{array}$ & (. $1.88-2.16)$ \\
\hline 4 & $\begin{array}{l}2.31 \\
\quad(1.76-3.01)^{* * *}\end{array}$ & $\begin{array}{l}1.71 \\
\quad\left(1.29^{-2.27}\right) * * *\end{array}$ & $\begin{array}{l}2.03 \\
\quad(1.61-2.57)^{* * *}\end{array}$ & $\begin{array}{l}1.55^{\circ} \\
\quad(1.17-1.93)^{* *}\end{array}$ & $\begin{array}{l}1.4^{\circ} \\
(1.11-1.75)^{* *}\end{array}$ & $\begin{array}{l}1.20 \\
\quad(0.91-1.57)\end{array}$ & $\stackrel{2.47}{(1.63-3.76) * * *}$ & $\begin{array}{l}2.29 \\
\quad(1.49-3.53)^{* * *}\end{array}$ \\
\hline 5 & $\begin{array}{l}2.32 \\
\quad(1.84-2.92) * * *\end{array}$ & $\begin{array}{l}1.37 \\
\quad(0.99-1.89)\end{array}$ & $\begin{array}{l}2.81 \\
\quad(2.31-3.41)^{* * * *}\end{array}$ & $\begin{array}{l}1.76 \\
\quad(1.33-2.34)^{* * * *}\end{array}$ & $\begin{array}{l}2.96 \\
\quad(2.39-3.68) * * *\end{array}$ & $\begin{array}{l}1.62 \\
\quad(1.20-2.18) * * *\end{array}$ & $\begin{array}{l}8.81 \\
\quad(6.69-11.79)^{* * * *}\end{array}$ & $\begin{array}{l}6.55 \\
\left(4.4^{6}-9.62\right)^{* * * *}\end{array}$ \\
\hline 6 & $\begin{array}{l}5.26 \\
\quad(3.90-7.08)^{* * * *}\end{array}$ & $\frac{2.64}{(1.84-3.79)^{* * *}}$ & $\begin{array}{l}6.02 \\
\quad(4.54-7.99)^{* * * *}\end{array}$ & $\begin{array}{l}3.13 \\
\quad\left(2.24-4.3^{8}\right) * * *\end{array}$ & $\begin{array}{l}3.85 \\
\quad(2.77-5.35)^{* * * *}\end{array}$ & $\begin{array}{l}2.91 \\
\quad(1.90-4.46)^{* * * *}\end{array}$ & $\begin{array}{l}4^{2} .79 \\
\quad\left(3^{0.4} 4^{6-60.12}\right)^{* * *}\end{array}$ & $\begin{array}{l}27.65 \\
\quad(18.57-41.18)^{* * *}\end{array}$ \\
\hline
\end{tabular}

Notes: ADL: activities of daily living. OR: odds ratio. CI: confidence interval. Group 1: no/low loneliness and isolation. Group 2: moderate loneliness. Group 3: lives alone. Group 4: moderate isolation. Group 5: moderate loneliness, lives alone. Group 6: high loneliness and moderate isolation. All adjusted models are adjusted for age, sex (male/female), marital status (married or partner/single/divorced or separated), employment (employed/ unemployed/retired), qualifications (university or higher education/high school or college/no qualifications) and net non-pension wealth (top three quintiles/bottom two quintiles). 1. Reference group: no issues with ADLs ( $\mathrm{N}$ in fully adjusted model $=5,975$ ). 2. Reference group: excellent/ very good/good self-rated health $(\mathrm{N}$ in fully adjusted model $=6,090) \cdot 3$. Reference group: no chronic conditions $(\mathrm{N}$ in fully adjusted model $=6, \mathrm{o} 88)$. 4. Reference group: low/no depressive symptoms as indicated by a score of $<3$ on the Center for Epidemiological Studies Depression Scale ( $\mathrm{N}$ in fully adjusted model $=6,041)$.

Significance levels: $* p<0.05, * * p<0.01, * * * p<0.001$ 
When we re-ran the logistic regression analysis with the cut-off of 3 , all results remained significant at the same level as when the cut-off was 4 , even for unadjusted analyses (see Table B in the online supplementary material). Thus, using a cut-point of 4 produced comparable results to a cut-point of 3 .

\section{Discussion}

Using clustering methodologies on a large data-set, we have been able to uncover meaningful sub-groups of older people based on shared characteristics of loneliness, living alone and social isolation in a community population of English older adults. Our results indicate that existing typologies that take into account the potential overlap or independence of social isolation and loneliness, such as the groupings suggested by Andersson (1986), reflect the experience of older adults. However, our work develops this typology further by demonstrating the presence of groups characterised by a high prevalence of living alone. Our work also suggests that we need to differentiate between those experiencing loneliness or isolation, or in combination, and that the severity of those issues may also be important in defining different typologies, as suggested by Victor et al. (2000). Therefore, we suggest considering both the number of issues that people have (i.e. none, one issue independently, two or more issues in combination) and the severity of these issues (e.g. is loneliness moderate or severe in its intensity) will be important in defining different typologies in future work.

The largest single group identified within our sample did not experience social isolation, loneliness and did not live alone (Group 1). This group was mostly younger, married, wealthier, better educated and had fewer issues with health and wellbeing. This observation provides support to the idea that health and wellbeing are important correlates of loneliness and social isolation in older adults (Heinrich and Gullone 20o6; Luanaigh and Lawlor 2008). It is possible that increased community and social contact could have helped this group to maintain their health, however, it could also be the case that this group do not experience loneliness and social isolation because they do not have any health issues (Berkman and Glass 2000; Ferlander 2007). Future research could examine the direction of causality between maintenance of health and lack of loneliness/social isolation. There is evidence suggesting that loneliness and social isolation are risk factors for the development of serious illness (Valtorta et al. 2016b) and mortality (Elovainio et al. 2017; Holt-Lunstad et al. 2015; Steptoe et al. $\left.2013^{b}\right)$. However, evidence also suggests that poor health is also a risk factor for the development of loneliness (Victor et al. 2005). Thus, 
examining longitudinal relationships between health and loneliness, isolation and living alone could be important.

When creating typologies, we have suggested it could be important to differentiate between groups who experience an issue independently or in combination with another issue. We identified two groups who lived alone. One of these groups only experienced living alone (Group 3), whereas the other group experienced living alone with moderate loneliness (Group 5). Both groups that lived alone were older than all other groups and were more likely to be widowed. However, Group 5 also reported loneliness despite a similar demographic profile to Group 3. When compared to the reference group, Group 5 had a higher likelihood of reporting depressive symptoms and poorer health whereas for Group 3, after adjustment for confounders, there was no association between group membership and health. Depressive symptoms (Barg et al. 2006; Cacioppo et al. 20o6) and poorer health (Victor et al. 2005) have previously been pointed to as important issues strongly associated with loneliness. Thus, it is possible the poorer health and mental wellbeing of Group 5 could be why this group also experiences loneliness. Future longitudinal work could further unpick the causality of this association.

The identification of Group 3 was also of theoretical and policy/practice interest. Previous theoretical conceptualisations of loneliness and isolation have differentiated these concepts from living alone, which is simply a measure of the type of household in which a person lives (Victor et al. 200o). The social isolation index from which our indicators of social isolation used in this analysis were derived includes living alone (Shankar et al. 2011 ). However, the identification of Group 3 supports the presupposition of Victor et al. (2000) that living alone can be differentiated from loneliness and other indicators of social isolation. Further investigation of Group 3 could also be particularly interesting to determine why, in the face of many of the socio-demographic risk factors for experiencing social isolation and loneliness such as living alone, widowhood, being female and older age (Pinquart and Sorensen $2001 a$; Wenger et al. 1996), they are less likely to report being lonely or socially isolated.

We also identified two groups who experienced moderate loneliness (Group 2) and some isolation (Group 4) independently. Group 2 were broader similar to Group 1 in socio-demographic terms but had worse health outcomes. Previous work has indicated that loneliness is strongly associated with health status (Luanaigh and Lawlor 20o8; Victor et al. 2005), thus it is possible that poorer psychological and physical health explained the moderate loneliness that characterised Group 2. Group 4 was particularly notable as it had a higher proportion of males than all other groups. This could be because men are less likely to report 


\section{Kimberley J. Smith and Christina Victor}

(Pinquart and Sörensen 2001 b) or recognise (Borys and Perlman 1985) feelings of loneliness compared with women, or that they are more likely to experience social rather than emotional loneliness (Dahlberg and McKee 2014). Our work adds to pre-existing literature by indicating that gender may be an important factor to examine when separating out groups based on loneliness and social isolation.

We have suggested that alongside taking the broad threefold typologies into consideration, the severity of these experiences could be important in differentiating between groups. This is particularly evident for Group 6. This group had the most severe profile of loneliness and social isolation, and also had a high likelihood of living alone. This group had the most pronounced association with all indicators of physical and mental health. Previous work has indicated that loneliness and depression share a strong relationship (Barg et al. 20o6; Cacioppo et al. 20o6). Our work adds to this by showing, when compared with a group with low/no loneliness or isolation, that loneliness, living alone and social isolation are linked with depressive symptoms. Our work also suggests that as loneliness severity increases, the association with depressive symptoms increases, as hypothesised by Victor et al. (2000).

However, it is important to note that most groups who experienced either social isolation and/or loneliness were all more likely to have high depressive symptoms when compared with the group with no loneliness or isolation, even after we adjusted for socio-demographic variables. This indicates that mental wellbeing should be considered in all people who may be socially isolated or lonely. Past work indicates that loneliness and social isolation share different relationships with health outcomes (Cornwell and Waite 2009; Holwerda et al. 2013; Shankar et al. 2011; Steptoe et al. 2013a). Our work adds to the research in this field by showing that when compared with a group with no loneliness or isolation, any group who experiences loneliness and/or social isolation are more likely to report poor health. This work also indicates identifying groups with differing characteristics of loneliness and social isolation using datadriven methodologies could represent an interesting avenue to tell us more about how loneliness, social isolation and living alone are linked with health.

\section{Limitations and considerations for future work}

There are methodological caveats to our findings. When interpreting these results it is important to note that LCA is an exploratory technique, and that model fit is not absolute. Both the number of groups examined and the characteristics of the groups were determined using likelihood estimates. 
It should be noted that there was considerable within-group heterogeneity for many of the indicators we examined, so while groups were identified as having a high likelihood of exhibiting certain characteristics, this is not to say every single person within this group definitively exhibited these characteristics (just that they were the group they were most likely to belong to based on their profile). Future work could use LCA methodologies within different populations to determine whether the proposed broad typologies are generalisable to different populations and samples. A related consideration for interpretation is that these groups were uncovered in a specific cohort, and may not generalise to other populations or cultures.

Measurement issues should also be considered. Both the loneliness and social isolation indicators examined were short, and likely would not capture the full complexity of experiences of social isolation or loneliness. There is also increasing evidence suggesting that the chronicity and changing nature of loneliness and social isolation are important issues to consider (Dahlberg et al. 2015; Dykstra, Van Tilburg and de Jong Gierveld 2005; Victor and Bowling 2012), but we could not address them. Future research could address these limitations by re-running this analysis in a study with more comprehensive loneliness and isolation measures that take chronicity into account.

The indicators of social isolation used in this study have also been used by other researchers to operationalise differing theoretical constructs such as social connectedness (Cornwell and Waite 2009) and social detachment (Jivraj, Nazroo and Barnes 2012; Tomaszewski and Barnes 2008). Thus, while we have used these items as indicators of social isolation, they could be indicating social connectedness, social detachment or other related concepts such as social exclusion, which future research could try to disentangle. Furthermore, it is important to determine whether isolation was an active or passive process. Qualitative work suggests that one can differentiate between voluntary and involuntary loneliness (Dahlberg 2007), and it is plausible to assume these different types of loneliness/isolation will have different associations with health and wellbeing. The cross-sectional nature of this analysis means we cannot infer causality. It is plausible that there is a bidirectional association of loneliness, living alone and social isolation with health and wellbeing, and we will explore this in our future work.

\section{Conclusions and implications}

Using LCA we have been able to uncover different groups based on shared experiences of loneliness, social isolation and living alone, showing the different experiences older adults can have. This has important implications 
for the creation of typologies based on loneliness, social isolation and living alone. Our work indicates considering the number of issues that people have (i.e. none, one, two or more) and then the severity of these is important in defining different typologies. However, in order to make use of these typologies it is important to replicate these results in different samples. We have shown that different groups share different associations with health and wellbeing. This work indicates that the lived experience of loneliness, social isolation and living alone in older adults is complex, and that taking the number of issues and severity of issues into account will be important for researchers and clinicians working with groups of older adults who may be experiencing these issues. It also demonstrates that living alone is conceptually separate from loneliness and isolation, and has limited utility as a measure of these complex concepts.

\section{Supplementary material}

To view supplementary material for this article, please visit https://doi.org/ $10.1017 /$ So $144686 X_{1} 8000132$.

\section{References}

Alpass, F. M. and Neville, S. 2003. Loneliness, health and depression in older males. Aging $\mathcal{E}^{2}$ Mental Health, 7, 3, $212-6$.

Andersson, L. 1986. A model of estrangement-including a theoretical understanding of loneliness. Psychological Reports, 58, 3, 683-95.

$\mathrm{Au}, \mathrm{B}$., Smith, K. J., Gariépy, G. and Schmitz, N. 2015. The longitudinal associations between C-reactive protein and depressive symptoms: evidence from the English Longitudinal Study of Ageing (ELSA). International Journal of Geriatric Psychiatry, 3o, 9, 976-84.

Badawi, G., Pagé, V., Smith, K.J., Gariépy, G., Malla, A., Wang, J., Boyer, R., Strychar, I., Lesage, A. and Schmitz, N. 2013. Self-rated health: a predictor for the three year incidence of major depression in individuals with Type II diabetes. Journal of Affective Disorders, 145, 1, 100-5.

Barg, F. K., Huss-Ashmore, R., Wittink, M. N., Murray, G. F., Bogner, H. R. and Gallo, J.J. 2006. A mixed-methods approach to understanding loneliness and depression in older adults. Journals of Gerontology: Psychological Sciences and Social Sciences, 61B, 6, S329-39.

Bassuk, S. S., Glass, T. A. and Berkman, L. F. 1999. Social disengagement and incident cognitive decline in community-dwelling elderly persons. Annals of Internal Medicine, 131, 3, 165-73.

Berkman, L. F. and Glass, T. 200o. Social integration, social networks, social support, and health. In Berkman, L. F. and Kawachi, I. (eds), Social Epidemiology. Oxford University Press, Oxford, 137-73.

Borys, S. and Perlman, D. 1985. Gender differences in loneliness. Personality and Social Psychology Bulletin, 1 1, 1, 63-74. 
Burholt, V., Dobbs, C. and Victor, C. 2017 . Social support networks of older migrants in England and Wales: the role of collectivist culture. Ageing $\mathcal{E}^{2}$ Society, 38, 7, $1453^{-}$ 77 .

Cacioppo, J. T. and Hawkley, L. C. 20o9. Perceived social isolation and cognition. Trends in Cognitive Sciences, 13, 10, 447-54.

Cacioppo, J. T., Hughes, M. E., Waite, L. J., Hawkley, L. C. and Thisted, R. A. 2006. Loneliness as a specific risk factor for depressive symptoms: cross-sectional and longitudinal analyses. Psychology and Aging, 2 1, 1, 140-51.

Cloutier-Fisher, D., Kobayashi, K. and Smith, A. 2011 . The subjective dimension of social isolation: a qualitative investigation of older adults' experiences in small social support networks. Journal of Aging Studies, 25, 4, 407-14.

Cornwell, E.Y. and Waite, L.J. 2009. Social disconnectedness, perceived isolation, and health among older adults. Journal of Health and Social Behavior, 5o, 1, 31-48.

Dahlberg, K. 2007. The enigmatic phenomenon of loneliness. International Journal of Qualitative Studies on Health and Well-being, 2, 4, 195-207.

Dahlberg, L., Andersson, L., McKee, K. J. and Lennartsson, C. 2015. Predictors of loneliness among older women and men in Sweden: a national longitudinal study. Aging E Mental Health, 19, 5, 409-17.

Dahlberg, L. and McKee, K. J. 2014 . Correlates of social and emotional loneliness in older people: evidence from an English community study. Aging $\mathcal{E}$ Mental Health, $18,4,5^{0} 4^{-1} 4$.

Davis, M., Murphy, S., Neuhaus, J. and Lein, D. 1990. Living arrangements and dietary quality of older US adults. Journal of the American Dietetic Association, 9o, $12,1667-72$.

Dean, A., Kolody, B., Wood, P. and Matt, G. E. 1992. The influence of living alone on depression in elderly persons. Journal of Aging and Health, 4, 1, 3-18.

Dhalwani, N. N., O’Donovan, G., Zaccardi, F., Hamer, M., Yates, T., Davies, M. and Khunti, K. 2016. Long terms trends of multimorbidity and association with physical activity in older English population. International Journal of Behavioral Nutrition and Physical Activity, $\mathbf{1 3}, 1,8$.

Dhalwani, N. N., Zaccardi, F., O’Donovan, G., Carter, P., Hamer, M., Yates, T., Davies, M. and Khunti, K. 2017. Association between lifestyle factors and the incidence of multimorbidity in an older English population. Journals of Gerontology, 72A, 4, 528-34.

Dykstra, P. A., Van Tilburg, T. G. and de Jong Gierveld, J. 2005. Changes in older adult loneliness: results from a seven-year longitudinal study. Research on Aging, $\mathbf{2 7}, 6,725-47$.

Ellwardt, L., Aartsen, M. and van Tilburg, T. 2016. Types of non-kin networks and their association with survival in late adulthood: a Latent Class Approach. Journals of Gerontology: Psychological Sciences and Social Sciences, 72B, 4, 694-705.

Elovainio, M., Hakulinen, C., Pulkki-Råback, L., Virtanen, M., Josefsson, K., Jokela, M., Vahtera, J. and Kivimäki, M. 2017. Contribution of risk factors to excess mortality in isolated and lonely individuals: an analysis of data from the UK Biobank cohort study. The Lancet Public Health, 2, 6, e26o-6.

Ferlander, S. 2007. The importance of different forms of social capital for health. Acta Sociologica, 5o, 2, $115^{-28 .}$

Gale, C. R., Cooper, C. and Aihie Sayer, A. 2015 . Prevalence of frailty and disability: findings from the English Longitudinal Study of Ageing. Age and Ageing, 44, 1, $162-5$.

Golden, J., Conroy, R. M., Bruce, I., Denihan, A., Greene, E., Kirby, M. and Lawlor, B. A. 2009. Loneliness, social support networks, mood and wellbeing in 
community-dwelling elderly. International Journal of Geriatric Psychiatry, 24, 7, $694-700$.

Hagenaars, J.A. and McCutcheon, A. L. 2002. Applied Latent Class Analysis. Cambridge University Press, Cambridge.

Hamer, M., Molloy, G. J., de Oliveira, C. and Demakakos, P. 20og. Leisure time physical activity, risk of depressive symptoms, and inflammatory mediators: the English Longitudinal Study of Ageing. Psychoneuroendocrinology, 34, 7, $10^{\mathrm{O}}{ }^{-} 5$.

Hawkley, L. C. and Cacioppo, J. T. 2010. Loneliness matters: a theoretical and empirical review of consequences and mechanisms. Annals of Behavioral Medicine, 4o, 2, $218-27$.

Hawthorne, G. 2008. Perceived social isolation in a community sample: its prevalence and correlates with aspects of peoples' lives. Social Psychiatry and Psychiatric Epidemiology, 43, 2, 140-50.

Heinrich, L. M. and Gullone, E. 20o6. The clinical significance of loneliness: a literature review. Clinical Psychology Review, 26, 6, 695-718.

Holt-Lunstad, J., Smith, T. B., Baker, M., Harris, T. and Stephenson, D. 2015. Loneliness and social isolation as risk factors for mortality: a meta-analytic review. Perspectives on Psychological Science, 10, 2, 227-37.

Holwerda, T. J., Deeg, D. J., Beekman, A. T., van Tilburg, T. G., Stek, M. L., Jonker, C. and Schoevers, R. A. 2013. Feelings of loneliness, but not social isolation, predict dementia onset: results from the Amsterdam Study of the Elderly (AMSTEL). Journal of Neurology, Neurosurgery, and Psychiatry, 85, $133-4$.

Hughes, M. E., Waite, L. J., Hawkley, L. C. and Cacioppo, J. T. 2004. A short scale for measuring loneliness in large surveys: results from two population-based studies. Research on Aging, 26, 6, 655-72.

Iliffe, S., Kharicha, K., Harari, D., Swift, C., Gillmann, G. and Stuck, A. E. 2007. Health risk appraisal in older people 2: the implications for clinicians and commissioners of social isolation risk in older people. British Journal of General Practice, 57, $537,277-82$.

Jivraj, S., Nazroo, J. and Barnes, M. 201 2. Change in social detachment in older age in England. In Banks, J., Nazroo, J. and Steptoe, A. (eds), The Dynamics of Ageing: Evidence from the English Longitudinal Study of Ageing 2002-10 (Wave 5). Institute for Fiscal Studies, London, 49-97.

Kharicha, K., Iliffe, S., Harari, D., Swift, C., Gillmann, G. and Stuck, A. E. 2007. Health risk appraisal in older people 1: are older people living alone an 'atrisk'group? British Journal of General Practice, 57, 537, 271-6.

Larson, R., Zuzanek, J. and Mannell, R. 1985. Being alone versus being with people: disengagement in the daily experience of older adults. Journal of Gerontology, 4o, 3 , $375^{-81}$.

Lee, H.Y., Jang, S.-N., Lee, S., Cho, S.-I. and Park, E.-O. 2008. The relationship between social participation and self-rated health by sex and age: a cross-sectional survey. International Journal of Nursing Studies, 45, 7, $1042-54$.

Luanaigh, C. Ó. and Lawlor, B. A. 2008. Loneliness and the health of older people. International Journal of Geriatric Psychiatry, 23, 12, $1213^{-2} 1$.

Lubben, J. and Gironda, M. 2003. Centrality of social ties to the health and wellbeing of older adults. In Berkman, B. and Harootyan, L. K. (eds), Social Work and Health Care in an Aging Society: Education, Policy, Practice and Research. Springer, New York, 319-50.

McCutcheon, A. L. 1987. Latent Class Analysis. Sage, Newbury Park, California.

McHugh, J., Kenny, R., Lawlor, B., Steptoe, A. and Kee, F. 201 7. The discrepancy between social isolation and loneliness as a clinically meaningful metric: 
findings from the Irish and English longitudinal studies of ageing (TILDA and ELSA). International Journal of Geriatric Psychiatry, 32, 6, 664-74.

Nummela, O., Seppänen, M. and Uutela, A. 2011. The effect of loneliness and change in loneliness on self-rated health (SRH): a longitudinal study among aging people. Archives of Gerontology and Geriatrics, 53, 2, 163-7.

Orth-Gomér, K., Rosengren, A. and Wilhelmsen, L. 1993. Lack of social support and incidence of coronary heart disease in middle-aged Swedish men. Psychosomatic Medicine, 55, 1, 37-43.

Perissinotto, C. M., Cenzer, I. S. and Covinsky, K. E. 2012. Loneliness in older persons: a predictor of functional decline and death. Archives of Internal Medicine, 172, 14, 1078-84.

Perissinotto, C. M. and Covinsky, K. E. 2014. Living alone, socially isolated or lonelywhat are we measuring? Journal of General Internal Medicine, 29, 1 1, 1429-31.

Pinquart, M. and Sorensen, S. $2001 a$. Influences on loneliness in older adults: a meta-analysis. Basic and Applied Social Psychology, 23, 4, 245-66.

Pinquart, M. and Sörensen, S. $2001 \mathrm{~b}$. Gender differences in self-concept and psychological well-being in old age: a meta-analysis. Journals of Gerontology: Psychological Sciences and Social Sciences, 56B, 4, 195-213.

Rook, K. S. 1984. The negative side of social interaction: impact on psychological well-being. Journal of Personality and Social Psychology, 46, 5, 1097-1 108.

Shankar, A., Hamer, M., McMunn, A. and Steptoe, A. 2013. Social isolation and loneliness: relationships with cognitive function during 4 years of follow-up in the English Longitudinal Study of Ageing. Psychosomatic Medicine, 75, 2, 161-70.

Shankar, A., McMunn, A., Banks, J. and Steptoe, A. 2011 . Loneliness, social isolation, and behavioral and biological health indicators in older adults. Health Psychology, 3o, $4,377-85$.

Steptoe, A., Breeze, E., Banks, J. and Nazroo, J. $2013 a$. Cohort profile: the English Longitudinal Study of Ageing. International Journal of Epidemiology, 42, 6, 1640-8.

Steptoe, A., Shankar, A., Demakakos, P. and Wardle, J. $2013 b$. Social isolation, loneliness, and all-cause mortality in older men and women. Proceedings of the National Academy of Sciences of the United States of America, 110, 15, 5797-801.

Sundström, G., Fransson, E., Malmberg, B. and Davey, A. 2009. Loneliness among older Europeans. European Journal of Ageing, 6, 4, 267-75.

Tomaszewski, W. and Barnes, M. 2008. Investigating the dynamics of social detachment in older age. In Banks, J., Breeze, E., Lessof, C. and Nazroo, J. (eds), Living in the 2 Ist Century: Older People in England. The Institute of Fiscal Studies, London, $15^{\mathrm{O}-85}$.

Townsend, P. 1980. Isolation, desolation and loneliness. In Shanas, E., Townsend, P., Wedderburn, D., Friis, H., Milhoj, P. and Stehouwer, J. (eds), Old People in Three Industrial Societies. Routledge \& Kegan Paul, London, $25^{8-87 .}$

Tunstall, J. 1966. Old and Alone: A Sociological Study of Old People. Routledge \& Kegan Paul, London.

Valtorta, N. and Hanratty, B. 2012. Loneliness, isolation and the health of older adults: do we need a new research agenda? Journal of the Royal Society of Medicine, $105,12,5^{18-22 .}$

Valtorta, N. K., Kanaan, M., Gilbody, S. and Hanratty, B. $2016 a$. Loneliness, social isolation and social relationships: what are we measuring? A novel framework for classifying and comparing tools. BMJ Open, 6, 4, eo10799.

Valtorta, N. K., Kanaan, M., Gilbody, S., Ronzi, S. and Hanratty, B. $2016 b$. Loneliness and social isolation as risk factors for coronary heart disease and stroke: systematic review and meta-analysis of longitudinal observational studies. Heart, 102, 13, 1009-16. 


\section{Kimberley J. Smith and Christina Victor}

Vermunt, J. K. and Magidson, J. 2002. Latent class cluster analysis. In Hagenaars, J. and McCutcheon, A. (eds), Applied Latent Class Analysis. Cambridge University Press, Cambridge, 89-106.

Victor, C. R. and Bowling, A. 201 2. A longitudinal analysis of loneliness among older people in Great Britain. Journal of Psychology, 146, 3, 313-31.

Victor, C., Scambler, S., Bond, J. and Bowling, A. 200o. Being alone in later life: loneliness, social isolation and living alone. Reviews in Clinical Gerontology, 10, 4, 407-17.

Victor, C. R., Scambler, S. J., Bowling, A. and Bond, J. 2005. The prevalence of, and risk factors for, loneliness in later life: a survey of older people in Great Britain. Ageing $\mathcal{E}$ Society, 25, 6, 357-75.

Walton, C. G., Shultz, C. M., Beck, C. M. and Walls, R. C. 1991. Psychological correlates of loneliness in the older adult. Archives of Psychiatric Nursing, 5, 3, 165-70.

Weiss, R. S. 1973. Loneliness: The Experience of Emotional and Social Isolation. MIT Press, Cambridge, Massachusetts.

Wenger, G. C., Davies, R., Shahtahmasebi, S. and Scott, A. 1996. Social isolation and loneliness in old age: review and model refinement. Ageing $\mathcal{E}$ Society, 16, 3, $333^{-} 5^{8}$.

Zebhauser, A., Hofmann-Xu, L., Baumert, J., Häfner, S., Lacruz, M., Emeny, R., Döring, A., Grill, E., Huber, D. and Peters, A. 2014. How much does it hurt to be lonely? Mental and physical differences between older men and women in the KORA-Age Study. International Journal of Geriatric Psychiatry, 29, 3, 245 $5^{2}$.

Zunzunegui, M.-V., Alvarado, B. E., Del Ser, T. and Otero, A. 2003. Social networks, social integration, and social engagement determine cognitive decline in community-dwelling Spanish older adults. Journals of Gerontology: Psychological Sciences and Social Sciences, 58B, 2, S93-100.

Accepted 3 I January 20I8; first published online Io April 2018

Address for correspondence:

Kimberley J. Smith,

University of Surrey,

Stag Hill Campus,

Guildford GU2 7 XH, UK

E-mail: Kimberley.j.smith@surrey.ac.uk 